\title{
Prognostic Impact of Carcinoembryonic Angtigen (CEA) in Locally Advanced Cervical Cancer Treated with Concurrent Chemoradiotherapy
}

\author{
Kiyosumi Shibata*, Hiroaki Kajiyama, Mik Mizuno, Eiko Yamamoto, Fumitaka Kikkawa \\ Department of Obstetrics and Gynecology, Graduate School of Medicine, Nagoya University, Nagoya, Japan \\ Email: "shiba@med.nagoya-u.ac.jp
}

Received August 24, 2012; revised September 27, 2012; accepted October 8, 2012

\begin{abstract}
Objective: To identify prognostic factors in patients with locally advanced cervical cancer treated with concurrent chemoradiotherapy (CCRT). Methods and Materials: We analyzed 76 patients with FIGO stage IB2-IVb cervical cancer treated with CCRT between 2001 and 2006 at the Nagoya University Hospital. Patients with an advanced cervical cancer treated with CCRT. Overall survival (OS) and Progression-free survival (PFS) rates were estimated using the Kaplan-Meier method. The log-rank test was used to test differences in survival. Fisher's exact test was employed for univariate analysis. The Cox proportional hazard model was used for multivariate analysis. Results: The median age was 52, and the median follow-up period was 36 months. The 5-year OS and PFS rates of all patients were $88.2 \%$ and $72.4 \%$, respectively. Twenty-one of the 76 patients were diagnosed with recurrence. A higher serum CEA before CCRT was an independent predictive factor for a poor prognosis on multivariate analysis. Conclusions: A high level of serum CEA was a predictive factor for a poor prognosis. New strategies should be considered to control disease in this group of patients.
\end{abstract}

Keywords: Cervical Cancer; Chemoradiotherapy; Recurrence; Prognostic Factor; Carcinoembryonic Angtigen (CEA)

\section{Introduction}

Cervical cancer is a significant cause of death from malignant disease in women in developing countries, and most of these cases have locally advanced at the time of diagnosis [1]. Both radical hysterectomy with lymphadenectomy and radiation therapy are used for advanced cervical cancer. However, the survival rate in women with advanced cervical cancer has remained relatively unchanged over the last two decades. Most investigators agree that the cervical tumor size is a significant negative prognostic factor, since bulky tumors are associated with a high incidence of lymph node metastases as well as recurrence as compared with smaller tumors $[2,3]$. Recurrence is more frequent in patients with the involvement of lymph nodes, parametrium, and surgical margins, and in patients with large or deeply invasive lesions. Recently, four randomized studies, including almost 2000 patients, showed that the concomitant administration of cisplatin-based chemotherapy and radiation could significantly improve survival as compared with the conventional use of radiotherapy as exclusive therapy [4-7]. It is therefore important to determine fac-

${ }^{*}$ Corresponding author. tors that indicating a poor prognosis. Various predictive factors for recurrence in advanced cervical cancer have been reported, such as lymph node metastasis, the tumor size, and hemoglobin level. Here in, we retrospectively analyzed 76 patients with locally advanced cervical cancer treated with CCRT in our institution, and investigated factors indicating a poor prognosis.

\section{Patients and Methods}

We retrospectively analyzed 76 patients with squamous cell carcinoma and adenocarcinoma of the uterine cervix who were treated with CCRT between 2001 and 2006 at the University of the Nagoya Hospital. The clinical staging was based on FIGO stage classifications. The procedure for staging included a detailed history and a physical examination, as well as common laboratory tests and standard chest radiographs, intravenous pyelograms, Xrays, cystoscopies, and sigmoidoscopies. In the evaluation of lymph node involvement, computed tomography (CT) scans or magnetic resonance imaging (MRI) were performed in all patients. The principal criterion for positive node involvement was based on the axial diameter of the lymph node. Lymph nodes larger than $1 \mathrm{~cm}$ in the short-axis dimension were considered abnormal. All 
patients gave written informed consent for the treatment. The study design was described in our previous report [8]. The schedule of concomitant chemotherapy was as follows: intraarterial or intravenous infusion of $70 \mathrm{mg} \cdot \mathrm{m}^{2}$ cisplatin, days 1 and 22; 24-hour continuous intravenous infusion of $700 \mathrm{mg} \cdot \mathrm{m}^{2} 5$-FU, days $1-4$ and $22-25$. The intravenous infusion of cisplatin was performed only in patients suspected of having lymph node metastasis by computed tomography (CT) or MRI. For intraarterial infusion, a catheter was introduced via the femoral artery using Seldinger's technique and the tip was advanced to a level beyond the superior gluteal artery, a branch of the internal iliac artery, or as far as the uterine artery. After confirming the blood supply to the uterus by angiography, $70 \mathrm{mg} \cdot \mathrm{m}^{2}$ cisplatin $\left(35 \mathrm{mg} \cdot \mathrm{m}^{2}\right.$ each into the left and right uterine arteries) was infused over five minutes.

Two weeks after the end of concurrent chemoradiation, patients were restaged and evaluated regarding the objective response and operability, based on a second MRI and clinical examination. The response to CCRT was defined as follows: a complete response (CR) indicated the disappearance of all measurable disease; a partial response (PR) was a $50 \%$ or greater reduction in the product of the transverse diameters of the cervical lesions; stable disease (SD) was a reduction $<50 \%$ or an increase $<25 \%$ in the product of the transverse diameters of the cervical lesions; progressive disease (PD) was a $25 \%$ or greater increase in the product of the transverse diameters of the cervical lesions. Toxicity assessment was performed according to the World Health Organization criteria.

Four weeks after the end of CCRT, the patients underwent surgery. Regarding the surgery types, radical hysterectomy and pelvic lymphadenectomy were performed when applicable. When the surgeon judged that radical hysterectomy was not applicable because of the state of residual tumors and complications, modified radical hysterectomy or simple total hysterectomy was selected. The surgical specimen was carefully examined to assess the following parameters: macroscopic residual disease, microscopic residual disease, parametrial involvement, vaginal involvement, lymph node involvement, and lymph vascular space involvement. Patients with tumors involving the surgical margins were referred for additional chemotherapy. Patients were followed-up every 3 months with complete pelvic examinations, as well as blood counts, clinical chemistry, and chest Xrays. CT, ultrasound, and other studies were carried out when appropriate. Postoperative complications were also evaluated. Overall survival (OS) was defined as the time from the start of CCRT until death. Disease-free survival (DFS) was measured from the start of CCRT to the time of relapse. Survival was calculated according to the method of Kaplan and Meier, and a log-rank test was used to determine the significance of differences in the survival distribution.

The prognostic significance of several clinical and pathological variables was assessed using the multivariate Cox's proportional hazard's analysis. Stat View software ver.5.0 (SAS, Institution Inc., Cary, NC, USA) was used for all statistical analyses, and a p-value of $<$ 0.05 was considered significant.

\section{Results}

Patient characteristics are shown in Table 1. The median follow-up period was 36 months (range: 14 - 57 months). Staging of the disease was determined according to the International Federation of Gynecology and Obstetrics (FIGO) classification. Stages were distributed as follows: 11 patients in IB2, 3 patients in IIA, 38 patients in IIB, 2 patient in IIIA, 9 patients in IIIB, 6 patients in IVA, and 7 patients in IVB. All patients were available for the measurement of the response to treatment. A complete response was defined as no viable cancer cells clinically or pathologically at 3 months after the completion of CCRT. Complete responses were achieved in 55 of 76 patients $(72.4 \%)$ clinically. Fifty-five of the $76(72.4 \%)$ treated cases underwent surgery. Radical hysterectomy was performed in 26 patients, whereas 17 and 8 patients underwent modified radical and simple hysterectomy, respectively. Pelvic lymphadenectomy was performed in 52 patients. Twenty-one patients were positive for lymph node metastasis. The 5-year OS and PFS rates of all patients were $88.2 \%$ and $72.4 \%$, respectively (Figure 1). Relapse of disease was observed in 21 patients, distributed as follows: 6 central pelvic, 5 lymph nodal $(1 \mathrm{com}$ mon iliac and 4 aortic), 4 abdominal, 2 vaginal wall, and 4 lung metastases. The prognostic model for OS is based on 11 factors: age $50<$ years (vs. age $<50$ years), stage III disease (vs. stage III-IV disease), procedure to use CDDP (IA vs. IV), the tumor size $(<4 \mathrm{~cm}$ vs. $4 \mathrm{~cm}<$ ), pathological group (squamous cell carcinoma vs. adenocarcinoma), lymph node swelling (positive vs. negative), $\mathrm{Hb}(<8 \mathrm{~g} / \mathrm{dl}$ vs. $8 \mathrm{~g} / \mathrm{dl})$, serum CA125 level $(<35 \mathrm{U} / \mathrm{ml}$ vs. $35 \mathrm{U} / \mathrm{ml}<)$, serum SCC level $(<7 \mathrm{U} / \mathrm{ml}$ vs. $7 \mathrm{U} / \mathrm{ml}<)$, and serum CEA level $(<7 \mathrm{U} / \mathrm{ml}$ vs. $7 \mathrm{U} / \mathrm{ml}<)$. On univariate analysis of OS, an advanced stage, positive for lymph node swelling, and a higher serum CA125 level were independent predictors for a poorer survival (Table 2). On multivariate analysis of OS, no factor was an independent predictor for a poorer survival (Table 3). On univariate analysis of PFS, an advanced stage, squamous cell carcinoma, positive for lymph node swelling, a higher serum CA125 level, and higher serum CEA level were independent predictors for a poorer survival (Table 4). On multivariate analysis of PFS, only a higher serum CEA level $(p<0.05)$ was an independent predictor for a poorer survival (Table 5). The hazard ratio of a poor 
Table 1. Patient characteristics.

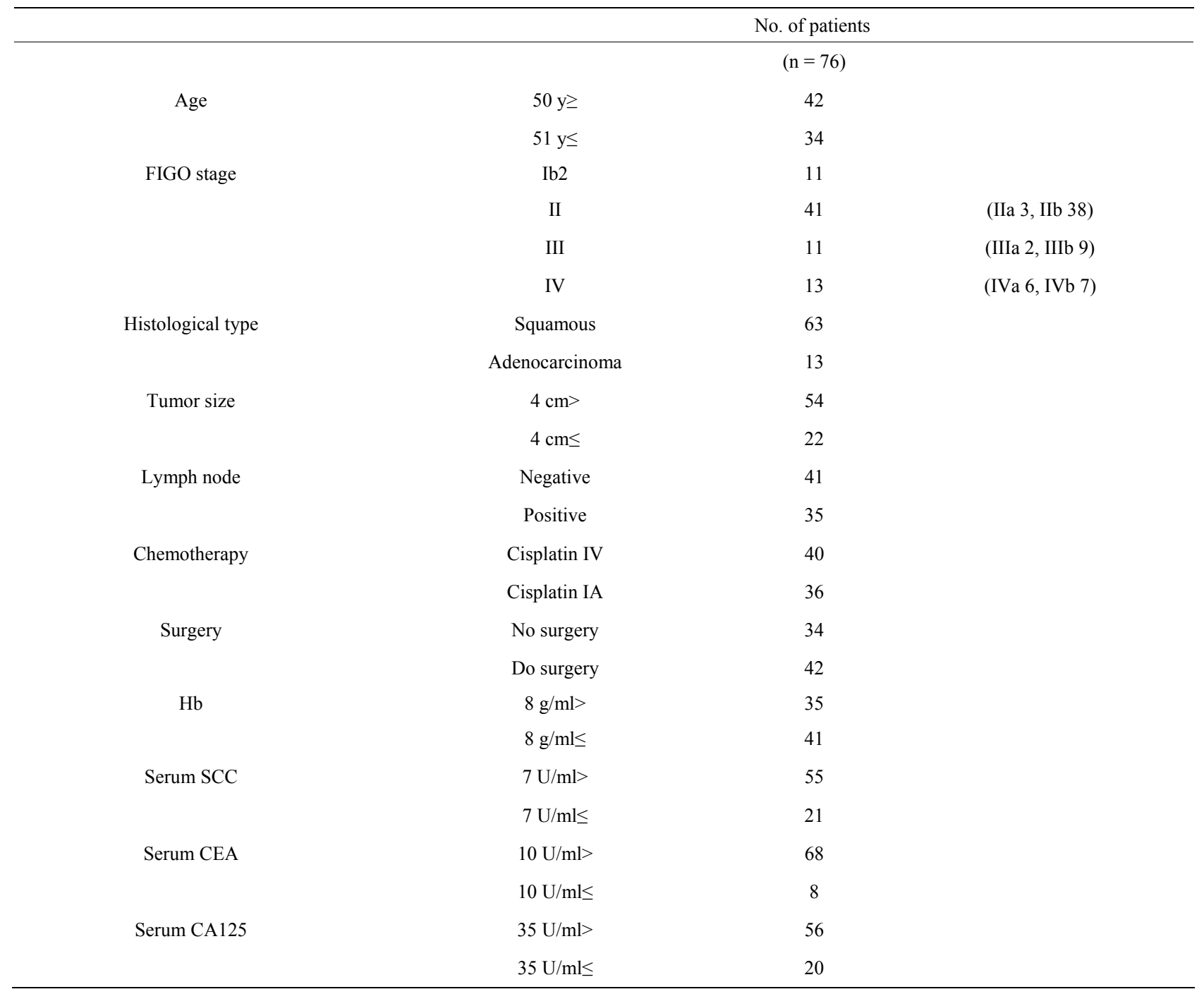

Table 2. Univariate analysis of overall survival.

\begin{tabular}{|c|c|c|c|}
\hline & Hazard ratio & $95 \%$ Confidence interval & p-value \\
\hline Age $(50 \mathrm{y} \geq$ vs. $51 \mathrm{y} \leq)$ & 6.619 & $0.756-50.323$ & 0.0893 \\
\hline Surgery (Do surgery vs. no surgery) & 0.749 & $0.187-3.001$ & 0.6828 \\
\hline Chemotherapy (cisplatin IV vs. IA) & 6.401 & $0.781-52.458$ & 0.0837 \\
\hline FIGO stage (III-IV vs. I-II) & 6.141 & $1.232-30.615$ & 0.0268 \\
\hline Tumor size $(4 \mathrm{~cm}>$ vs. $4 \mathrm{~cm} \leq)$ & 2.828 & $0.347-23.041$ & 0.3315 \\
\hline Histological type (squamous vs. adenocarcinoma) & 0.386 & $0.092-1.626$ & 0.1945 \\
\hline Lymph node (positive vs. negative) & 9.307 & $1.142-75.841$ & 0.0372 \\
\hline $\mathrm{Hb}(8 \mathrm{~g} / \mathrm{ml}>$ vs. $8 \mathrm{~g} / \mathrm{ml} \leq)$ & 0.35 & $0.070-1.753$ & 0.2017 \\
\hline Serum CA125 ( $35 \mathrm{U} / \mathrm{ml}>$ vs. $35 \mathrm{U} / \mathrm{ml} \leq)$ & 8.913 & $1.793-4.299$ & 0.0075 \\
\hline Serum SCC $(7 \mathrm{U} / \mathrm{ml}>$ vs. $7 \mathrm{U} / \mathrm{ml} \leq)$ & 2.688 & $0.669-10.800$ & 0.1636 \\
\hline Serum CEA $(10 \mathrm{U} / \mathrm{ml}>$ vs. $10 \mathrm{U} / \mathrm{ml} \leq)$ & 5.612 & $1.321-23.836$ & 0.0941 \\
\hline
\end{tabular}


Table 3. Univariate analysis of progression-free survival.

\begin{tabular}{|c|c|c|c|}
\hline & Hazard ratio & $95 \%$ Confidence interval & $\mathrm{p}$-value \\
\hline Age $(50 \mathrm{y} \geq$ vs. $51 \mathrm{y} \leq)$ & 1.701 & $0.686-4.216$ & 0.2514 \\
\hline Surgery (Do surgery vs. no surgery) & 0.684 & $0.289-1.615$ & 0.3858 \\
\hline Chemotherapy (cisplatin IV vs. IA) & 2.144 & $0.864-5.320$ & 0.0988 \\
\hline Tumor size ( $4 \mathrm{~cm}>$ vs. $4 \mathrm{~cm} \leq)$ & 2.783 & $0.819-9.458$ & 0.1011 \\
\hline Histological type (squamous vs. adenocarcinoma) & 0.371 & $0.149-0.922$ & 0.0328 \\
\hline Lymph node (positive vs. negative) & 2.438 & $1.007-5.904$ & 0.0438 \\
\hline $\mathrm{Hb}(8 \mathrm{~g} / \mathrm{ml}>$ vs. $8 \mathrm{~g} / \mathrm{ml} \leq)$ & 0.566 & $0.238-1.347$ & 0.1986 \\
\hline Serum CA125 (35 U/ml> vs. $35 \mathrm{U} / \mathrm{ml} \leq)$ & 2.535 & $1.064-6.044$ & 0.0358 \\
\hline Serum SCC ( $7 \mathrm{U} / \mathrm{ml}>$ vs. $7 \mathrm{U} / \mathrm{ml} \leq)$ & 1.792 & $0.742-4.326$ & 0.1945 \\
\hline Serum CEA $(10 \mathrm{U} / \mathrm{ml}>$ vs. $10 \mathrm{U} / \mathrm{ml} \leq)$ & 4.307 & $1.653-11.221$ & 0.0028 \\
\hline
\end{tabular}

Table 4. Multivariate analysis of overall survival.

\begin{tabular}{|c|c|c|c|}
\hline & Hazard ratio & $95 \%$ Confidence interval & p-value \\
\hline Age $(50 \mathrm{y} \geq$ vs. $51 \mathrm{y} \leq)$ & 4.152 & $0.306-56.240$ & 0.2844 \\
\hline Chemotherapy (cisplatin IV vs. IA) & 2.893 & $0.163-51.443$ & 0.4625 \\
\hline FIGO stage (III-IV vs. I-II) & 3.27 & $0.353-30.250$ & 0.2699 \\
\hline Lymph node (positive vs. negative) & 6.64 & $0.587-75.084$ & 0.1261 \\
\hline Serum CA125 (35 U/ml > vs. $35 \mathrm{U} / \mathrm{ml} \leq)$ & 7.142 & $0.951-53.643$ & 0.056 \\
\hline Serum SCC (7 U/ml> vs. $7 \mathrm{U} / \mathrm{ml} \leq)$ & 1.993 & $0.277-14.345$ & 0.4934 \\
\hline Serum CEA $(10 \mathrm{U} / \mathrm{ml}>$ vs. $10 \mathrm{U} / \mathrm{ml} \leq)$ & 2.583 & $0.276-24.130$ & 0.5042 \\
\hline
\end{tabular}

Table 5. Multivariate analysis of progression-free survival.

\begin{tabular}{|c|c|c|c|}
\hline & Hazard ratio & $95 \%$ Confidence interval & p-value \\
\hline Chemotherapy (cisplatin IV vs. IA) & 1.385 & $0.418-4.587$ & 0.5938 \\
\hline FIGO stage (III-IV vs. I-II) & 2.077 & $0.713-6.050$ & 0.1801 \\
\hline Tumor size $(4 \mathrm{~cm}>$ vs. $4 \mathrm{~cm} \leq)$ & 1.611 & $0.374-6.949$ & 0.5224 \\
\hline Lymph node (positive vs. negative) & 1.148 & $0.354-3.724$ & 0.8182 \\
\hline $\mathrm{Hb}(8 \mathrm{~g} / \mathrm{ml}>$ vs. $8 \mathrm{~g} / \mathrm{ml} \leq)$ & 1.243 & $0.394-3.923$ & 0.7108 \\
\hline Serum CA125 (35 U/ml> vs. $35 \mathrm{U} / \mathrm{ml} \leq)$ & 1.389 & $0.467-4.135$ & 0.5544 \\
\hline Serum SCC ( $7 \mathrm{U} / \mathrm{ml}>$ vs. $7 \mathrm{U} / \mathrm{ml} \leq)$ & 1.83 & $0.612-5.476$ & 0.2798 \\
\hline Serum CEA $(10 \mathrm{U} / \mathrm{ml}>$ vs. $10 \mathrm{U} / \mathrm{ml} \leq)$ & 3.349 & $1.185-9.468$ & 0.0226 \\
\hline
\end{tabular}




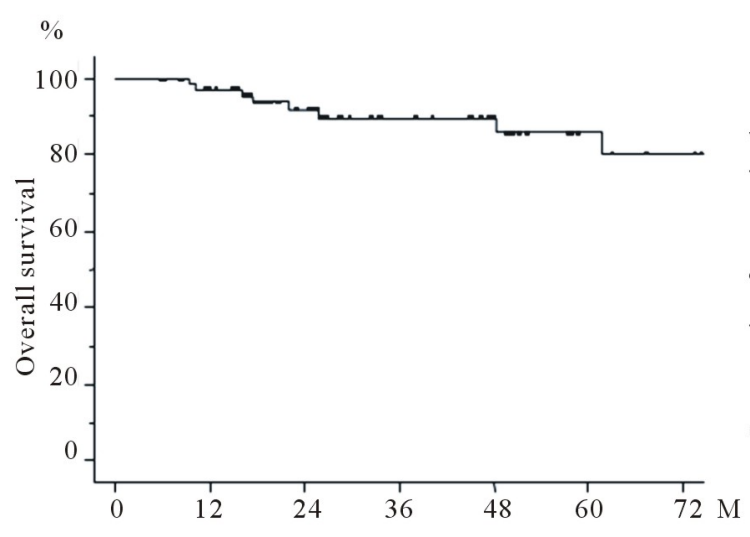

(a)

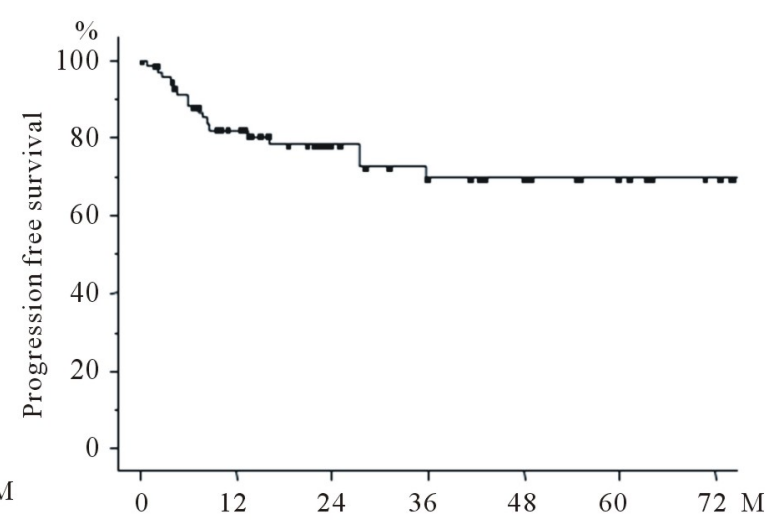

(b)

Figure 1. Overall survival (OS) and progression-free survival (PFS) curve drawn using Kaplan-Meier methods in 76 patients with locally advanced cervical cancer treated with CCRT. OS (a) and PFS (b) in all patients.

prognosis for patients with a higher serum CEA level was 3.49 (95\% confidence interval [CI], 1.185 - 9.468).

\section{Discussion}

We reported the effects of CCRT in our previous study. In this study, we increased the number of patients, and noted an increase in the response rate. We also investigated OS and PFS in this study, and the 5-year OS and PFS rates were $88.8 \%$ and $75 \%$, respectively. Considering that 10 patients in stages III and IV were included $(28 \%)$, the outcomes were better than those in the recently reported therapeutic results of advanced cervical carcinoma [9-11]. Furthermore, we performed surgery after CCRT in patients for whom it was applicable, and investigated the outcome after each surgical procedure. The 5-year PFS rates were $83.3 \%, 75 \%, 75 \%$, and $66.6 \%$ in the radical hysterectomy, modified radical hysterectomy, simple total hysterectomy, and non-surgical groups, respectively, showing no significant difference among the groups, although the result was slightly better in the radical hysterectomy group. Of 13 patients who were judged to show a CR and underwent surgery, a residual tumor was found in the surgical specimen after pathological examination in 4 patients $(31 \%)$. Of the 24 patients who underwent surgery, cancer infiltration in the parametrium was noted in 1 patient, and pelvic lymph node metastasis was noted in 7 patients $(29 \%)$. No severe postoperative complication occurred because surgery was performed after CCRT, but the incidence of complications was high in the radical hysterectomy group, and the incidences of bladder atony and lymphocyst were particularly high. The above findings suggested that, in our study, it was better to perform surgery after CCRT when it was applicable. Regarding the site of relapse, local and intrapelvic relapses were well controlled, but the control of relapse at distant sites was poor. Relapse occurred in 5 patients, including 3 in whom solitary paraaortic lymph node metastasis occurred. The prevention of distant relapse, mainly relapse in the paraaortic lymph node, is a future area of study. A previous report showed that paraaortic nodal metastasis is the most significant prognostic factor in patients with locally advanced cervical cancer [12]. For prevention measures, additional irradiation of the paraaortic lymph node region, and modification of the chemotherapy regimen and administration method, should be considered.

In this study, we found that high serum CA125 and CEA levels were useful factors to predict recurrence after preoperative CCRT. CEA levels were reported to be elevated at the time of recurrence, particularly in paraaortic lymph node metastasis [13]. A recent study reported the association of the CEA level with the response rate of preoperative CCRT [14]; however, this paper is the first to report an association between the CEA level and prognosis. In addition, a previous study reported that the serum CA125 level was a prognostic factor for cervical adenocarcinoma [15], which was the case in this study, in which the majority of patients had cervical squamous cell carcinoma. An association between CEA expression and 5-FU sensitivity has been reported, and our results also suggest an association between CEA and chemoradiation sensitivity, which requires further study. Although a previous study reported that SCC was a prognostic factor after CCRT [16], this was not the case in the present study. The difference in the results appears to be due to the fact that we used a combination of 5-FU-based CCRT and surgery.

Although several methods of chemotherapy for CCRT have been reported, the optimum combination and administration method have not been established. Preoperative CCRT improved the short-term prognosis of advanced cervical carcinoma. Additional investigations 
including long-term outcomes and new strategies should be considered to control disease in which serum CEA and CA125 are at high levels.

\section{Conclusion}

Our results suggest that a high level of serum CEA was a predictive factor for a poor prognosis. Thus, further prospective studies that include many cases are needed, and strategies should be considered to control disease in this group of patients.

\section{REFERENCES}

[1] M. Parkin, F. Bray, J. Ferlay and P. Pisani, "Global Cancer Statistics," CA: A Cancer Journal for Clinicians, Vol. 55, No. 2, 2005, pp. 74-108. doi:10.3322/canjclin.55.2.74

[2] L.-C. Horn, U. Fischer, G. Raptis, K. Bilek and B. Hentschel, "Tumor Size Is of Prognostic Value in Surgically Treated FIGO Stage II Cervical Cancer," Gynecologic Oncology, Vol. 107, No. 2, 2007, pp. 310-315. doi:10.1016/i.ygyno.2007.06.026

[3] C. A. Perez, P. W. Grigsby, K. S. Chao, D. G. Mutch and M. A. Lockett, "Tumor Size, Irradiation Dose, and LongTerm Outcome of Carcinoma of Uterine Cervix," International Journal of Radiation Oncology, Biology, Physics, Vol. 41, No. 2, 1998, pp. 307-317. doi:10.1016/S0360-3016(98)00067-4

[4] M. Morris, P. J. Eifel, J. Lu, et al., "Pelvic Radiation with Concurrent Chemotherapy Compared with Pelvic and Para-Aortic Radiation for High-Risk Cervical Cancer," The New England Journal of Medicine, Vol. 340, No. 15, 1999, pp. 1137-1143. doi:10.1056/NEJM199904153401501

[5] H. M. Keys, B. N. Bundy, F. B. Stehman, et al., "Cisplatin, Radiation, and Adjuvant Hysterectomy Compared with Radiation and Adjuvant Hysterectomy for Bulky Stage IB Cervical Carcinoma," The New England Journal of Medicine, Vol. 340, No. 15, 1999, pp. 1154-1161. doi:10.1056/NEJM199904153401503

[6] P. G. Rose, B. N. Bundy, E. B. Watkins, et al., "Concurrent Cisplatin-Based Radiotherapy and Chemotherapy for Locally Advanced Cervical Cancer," The New England Journal of Medicine, Vol. 340, No. 15, 1999, pp. 11441153. doi:10.1056/NEJM199904153401502

[7] W. A. Peters III, P. Y. Liu, R. Barrett, et al., "Concurrent Chemotherapy and Pelvic Radiation Therapy Compared with Pelvic Radiation Therapy Alone as Adjuvant Therapy after Radical Surgery in High-Risk Early-Stage Cancer of the Cervix," Journal of Clinical Oncology, Vol. 18, No. 8, 2000, pp. 1606-1613. doi:10.1097/00006254-200008000-00017

[8] K. Shibata, F. Kikkawa, Y. Suzuki, et al., "Usefulness of Preoperative Chemoradiation in Locally Advanced Cervical Carcinoma," Gynecologic and Obstetric Investigation, Vol. 57, No. 2, 2004, pp. 93-99. doi: $10.1159 / 000075385$

[9] C. W. Whitney, W. Sause, B. N. Bundy, et al., "A Randomized Comparison of Fluorouracil Plus Cisplatin versus Hydroxyurea as an Adjunct to Radiation Therapy in Stage IIB-IVA Carcinoma of the Cervix with Negative Para-Aortic Lymph Nodes: A Gynecologic Oncology Group and Southwest Oncology Group Study," Journal of Clinical Oncology, Vol. 17, No. 5, 1999, pp. 1339-1348.

[10] R. Pearcey, M. Brundage, P. Drouin, et al., "Phase III Trial Comparing Radical Radiotherapy with and without Cisplatin Chemotherapy in Patients with Advanced Squamous Cell Cancer of the Cervix," Journal of Clinical Oncology, Vol. 20, No. 4, 2002, pp. 966-972. doi:10.1200/JCO.20.4.966

[11] R. S. Lavey, P. Y. Liu, B. E. Greer, et al., "Recombinant Human Erythropoietin as an Adjunct to Radiation Therapy and Cisplatin for Stage IIB-IVA Carcinoma of the Cervix: A Southwest Oncology Group Study," Gynecologic Oncology, Vol. 95, No. 1, 2004, pp. 145-151. doi:10.1016/j.ygyno.2004.07.009

[12] P. W. Grigsby, M. L. Vest and C. A. Perez, "Recurrent Carcinoma of the Cervix Exclusively in the Paraaortic Nodes Following Radiation Therapy," International Journal of Radiation Oncology, Biology, Physics, Vol. 28, No. 2, 1994, pp. 451-455. doi:10.1016/0360-3016(94)90070-1

[13] H.-H. Chou, C.-C. Wang, C.-H. Lai, et al., "Isolated Paraaortic Lymph Node Recurrence after Definitive Irradiation for Cervical Carcinoma," International Journal of Radiation Oncology, Biology, Physics, Vol. 51, No. 2, 2001, pp. 442-448. doi:10.1016/S0360-3016(01)01628-5

[14] S. M. Yoon, K. H. Shin, J.-Y. Kim, et al., "The Clinical Values of Squamous Cell Carcinoma Antigen and Carcinoembryonic Antigen in Patients with Cervical Cancer Treated with Concurrent Chemoradiotherapy," International Journal of Gynecological Cancer, Vol. 17, No. 4, 2007, pp. 872-878. doi:10.1111/j.1525-1438.2007.00878.x

[15] A. Gadducci, R. Tana, A. Fanucchi and A. R. Genazzani, "Biochemical Prognostic Factors and Risk of Relapses in Patients with Cervical Cancer," Gynecologic Oncology, Vol. 107, No. 1, 2007, pp. S23-S26. doi:10.1016/j.ygyno.2007.07.033

[16] M. Hirakawa, Y. Nagai, M. Inamine, et al., "Predictive Factor of Distant Recurrence in Locally Advanced Squamous Cell Carcinoma of the Cervix Treated with Concurrent Chemoradiotherapy," Gynecologic Oncology, Vol. 108, No. 1, 2008, pp. 126-129.

doi:10.1016/j.ygyno.2007.08.091 